\title{
Role of Giant Dielectric Permittivity Effect during Tornado Generation Process
}

\author{
S.A. Maslov ${ }^{1,2}$, V.L. Natyaganov ${ }^{1}$ \\ ${ }^{1}$ Lomonosov Moscow State University, \\ Moscow, 119991, Russia \\ ${ }^{2}$ Joint Institute for High Temperatures of the RAS, \\ Moscow, 125412, Russia \\ sergm90@mail.ru, tenzor-home@yandex.ru
}

\begin{abstract}
Basing on electrohydrodynamics equations system, the article researches the role of electromagnetic factors with trigger nature in generating tornadoes: atmospheric electric field perturbations under thundercloud and giant dielectric permittivity effect of cloud as water droplets suspension with thin surface double electric layer. The work considers the influence of tripole cloud charge structure and effect of giant electric permittivity on forming tornado funnels and appearing number of accompanying specific phenomena: a "collar" in the funnel base, "devil horns" and growth of dust or water hill (cascade) toward the descending tornado funnel. Other factors (same or different direction of thundercloud rotation, distinguish of oxygen and nitrogen magnetic properties etc.) usually play secondary role in tornado-like vortices formation.
\end{abstract}

Keywords: atmospheric electric field, thundercloud, tornado, electrohydrodynamic pressure, giant dielectric permittivity effect.
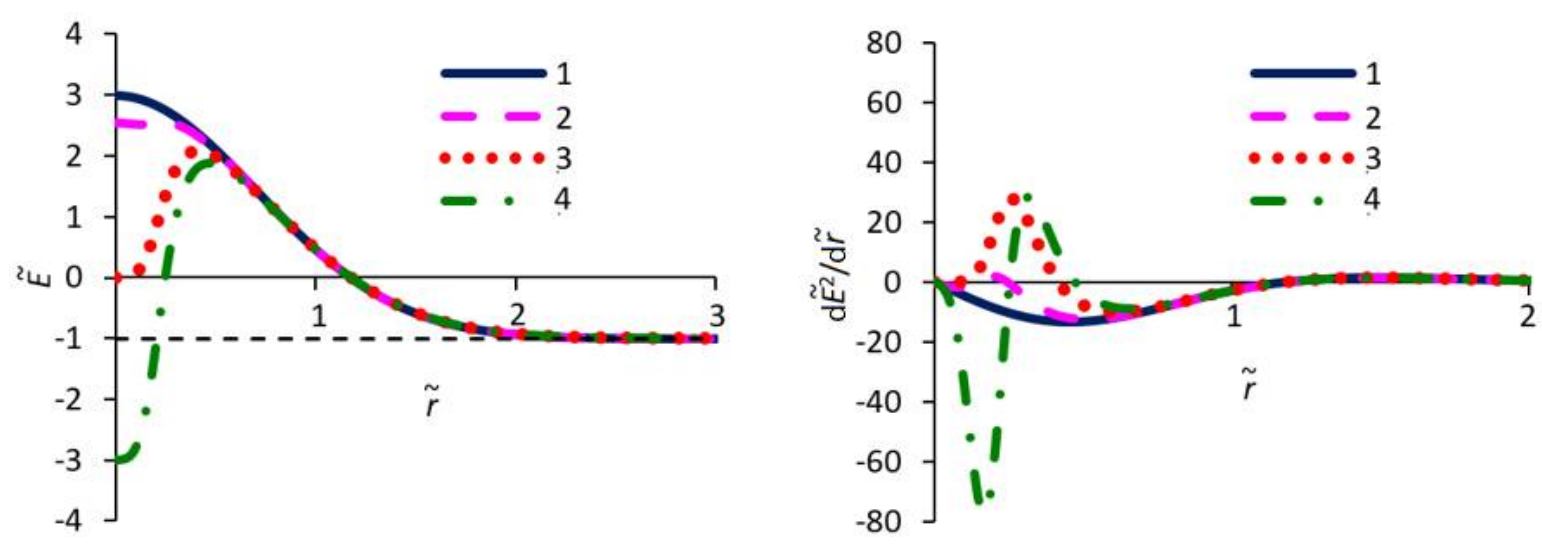

Graphs of atmospheric electic field perturbations (left) and gradient of their magnitude square (right) beneath a dipole thundercloud (1), at the initial stage of tripole electric structure formation (2), at the stage of appearing atmospheric electric field re-reversal (3) and beneath a mature tripole cloud (4).

$$
\begin{gathered}
\tilde{E}(\tilde{r})=\frac{E_{z}(\tilde{r})}{E_{0}}=\frac{(4 \tilde{r})^{4}+\beta}{(4 \tilde{r})^{4}+1}\left[N \cdot \exp \left(-\tilde{r}^{2}\right)-1\right] \\
\varepsilon_{\mathrm{e}} \approx \frac{c\left(\varepsilon_{\Sigma}-1\right) \overline{\left[\mathbf{u}_{\Sigma} \times \mathbf{B}_{\Sigma}\right]}}{\nabla\left\langle\Phi_{*}\right\rangle-c \overline{\nabla\langle\Phi\rangle}+c \overline{\nabla\left\langle\Phi^{\prime}\right\rangle}} \sim c\left(\varepsilon_{\Sigma}-1\right) q u_{0} u_{1} \frac{a}{d} \gg 1
\end{gathered}
$$




\title{
Роль эффекта гигантской диэлектрической проницаемости в процессе генерации торнадо
}

\author{
С.А. Маслов ${ }^{1,2}$, В.Л. Натяганов ${ }^{1}$ \\ ${ }^{1}$ Московский государственный университет имени М.В. Ломоносова, \\ Россия, Москва, 119991, Ленинские горы, д. 1 \\ ${ }^{2}$ Объединенный институт высоких температур РАН, \\ Россия, Москва, 125412, ул. Ижорская, д. 13 \\ sergm90@mail.ru, tenzor-home@yandex.ru
}

\begin{abstract}
Аннотация
На основе системы уравнений электрогидродинамики исследована роль электромагнитных факторов триггерного характера в генерации торнадо (смерчей) и струйных низовых прорывов: возмущений атмосферного электрического поля под грозовыми облаками и эффекта гигантской диэлектрической проницаемости облака как суспензии водяных микрокапель с тонким поверхностным двойным электрическим слоем. Рассмотрено совокупное влияние трипольной структуры облачного заряда и эффекта гигантской диэлектрической проницаемости на образование воронок торнадо и возникновение ряда сопутствующих специфических явлений: «воротника» в основании воронки, «рогов дьявола» и роста холма пыли или брызг (каскада) навстречу опускающейся воронке смерча. Другие факторы (одно- или разнонаправленная закрутка центра и периферии грозового облака, различие магнитных свойств атмосферного кислорода и азота и т.д.) обычно играют вспомогательную роль в формировании торнадоподобных вихрей.
\end{abstract}

Ключевые слова: атмосферное электрическое поле, грозовое облако, торнадо, электрогидродинамическое давление, эффект гигантской диэлектрической проницаемости.

\section{1. Введение}

В геофизической литературе гипотезы и модельные подходы к описанию торнадо (смерчей) условно разделяются на чисто гидродинамические, термогидродинамические и гидроэлектромагнитные. Гипотезы последнего типа появились намного раньше: М.В. Ломоносов (1753 г., Россия) первым выдвинул гипотезу об электрической природе торнадо [1], которая почти через столетие была поддержана Р. Хейром (1837 г., США) и Ж. Пельтье (1840 г., Франция). Лишь потом возникли чисто гидро- и термогидродинамические подходы к моделированию торнадо, а электрическая гипотеза была забыта вплоть до 1960-х гг.

В то же время некоторые характерные черты торнадо [2] не нашли адекватного обоснования в рамках гидро- и термогидродинамических подходов. Главные из них - высокая электрическая активность торнадо (частые вспышки молний и разнообразные свечения воронки); тесная связь смерча с материнским грозовым облаком; образование холма пыли или брызг еще до касания воронки с подстилающей поверхностью; формирование до сих пор загадочных престер-торнадо [3], опускающихся из облака в виде огненного столба и выжигающих траву и кустарники вплоть до корневой системы, о которых писал еще М.В. Ломоносов.

Поэтому в ряде работ вслед за [3] предлагаются различные гидроэлектромагнитные [4] подходы к изучению торнадогенеза. В частности, в работе [5] был показан определяющий характер электромагнитных факторов для модели воронки торнадо в развитой стадии и форме соосных конусов. В статьях $[1,6,7]$ была предпринята попытка обоснования важной 
роли электрогидродинамических (ЭГД) механизмов на начальном этапе формирования воронки торнадо до ее касания с подстилающей поверхностью с учетом сильных возмущений атмосферного электрического поля (АЭП) под материнским грозовым облаком.

В настоящей работе проводится сравнительный анализ влияния различных электромагнитных факторов на генерацию торнадо из грозовых облаков: величины и топологического вида сильных возмущений АЭП под облаком; эффекта гигантской диэлектрической проницаемости (ЭГДП) суспензии облачных капель с поверхностным зарядом тонкого двойного электрического слоя (ДЭС); возникающего электровихревого течения (ЭВТ) при перезарядке дипольного облака в трипольное; генерации магнитного поля при разных видах закрутки торнадо-циклона (ТЦ); различия магнитных свойств атмосферного кислорода и азота.

\section{2. Роль гигантской диэлектрической проницаемости грозового облака и распределения возмущений электрического поля под ним}

Грозовое облако в развитой стадии чаще всего имеет дипольную или трипольную структуру заряда [8-10]. В первом случае верхний слой облака обычно заряжен положительно, нижний - отрицательно. В трипольном облаке в центре нижней части располагается сравнительно малая область дополнительного положительного заряда, роль и механизмы формирования которой вызывали вопросы у многих исследователей [10]. Проанализируем роль зарядовой структуры облака с использованием системы ЭГД-уравнений $[11,12]$ :

$$
\begin{cases}\rho(\partial \mathbf{u} / \partial t+(\mathbf{u} \nabla) \mathbf{u})=-\nabla p_{\mathrm{e}}+\mu \Delta \mathbf{u}+\rho_{\mathrm{e}} \mathbf{E}, & \operatorname{div} \mathbf{u}=0, \\ p_{\mathrm{e}}=p-\varepsilon_{0}(\varepsilon-1) E^{2} / 2, \operatorname{div}\left(\varepsilon \varepsilon_{0} \mathbf{E}\right)=\rho_{\mathrm{e}}, & \operatorname{rot} \mathbf{E}=0,\end{cases}
$$

где $p_{\mathrm{e}}$ - ЭГД-давление; $\mathbf{E}$ - напряженность АЭП; $\varepsilon_{0}$ - диэлектрическая постоянная; $\varepsilon$ - относительная диэлектрическая проницаемость (ДП); $\rho_{\mathrm{e}}$ - зарядовая плотность, остальные обозначения стандартны для гидродинамики. С целью качественной аппроксимации возмущений вертикальной компоненты АЭП под облаком в [12] впервые предложена двухпараметрическая формула

$$
\tilde{E}(\tilde{r})=\frac{E_{z}(\tilde{r})}{E_{0}}=\frac{(4 \tilde{r})^{4}+\beta}{(4 \tilde{r})^{4}+1}\left[N \cdot \exp \left(-\tilde{r}^{2}\right)-1\right],
$$

где $E_{0} \sim 100 \mathrm{~B} / \mathrm{M}$ - поле «ясной погоды»; $\tilde{r}$ - безразмерное расстояние до оси облака. Параметр $N$ соответствует росту АЭП под грозовыми облаками, иногда до $10^{5} \div 10^{6} \mathrm{~B} / \mathrm{M}$, т.е. $N$ может составлять от нескольких единиц до $10^{3} \div 10^{4}$. Вариация параметра $\beta$ отвечает за изменение топологии возмущений АЭП при «перезарядке» облака [6] с дипольного $(\beta=1)$ на развитое трипольное $(\beta<0)$ с двойным реверсом [1] направления АЭП. На рис. 1 представлены профили $\tilde{E}(\tilde{r})$ при разных $\beta$, где для приемлемости вертикального масштаба выбрано $N=4$.

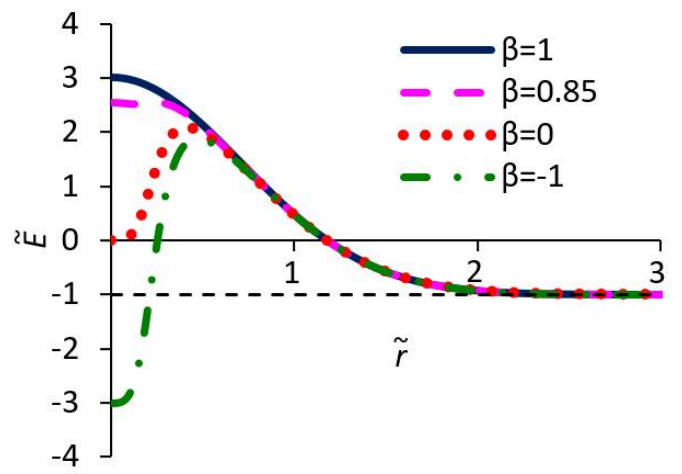

Рис. 1. Графики $\tilde{E}(\tilde{r})$ при $N=4$ и различных значениях $\beta$ 
Из (1), (2) видно, что под центром грозового облака за счет сильных возмущений АЭП при $N \gg 1$ существенно снижается ЭГД-давление $p_{\mathrm{e}}$, т.е. усиление электрического поля под центром грозового облака создает благоприятные условия для формирования торнадо и струйных низовых прорывов - downburst по терминологии [14].

Помимо сильных возмущений АЭП, важным триггерным фактором формирования тор-

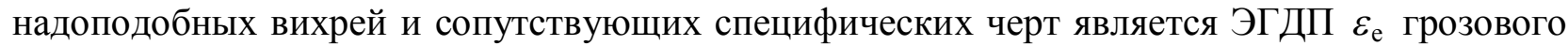
облака [15] как суспензии заряженных микрокапель воды с тонким ДЭС [8]. Этот неожиданный, но важный эффект можно объяснить на основе электромагнитного обобщения [16] классической задачи [17] об электрокапиллярном движении (ЭКД) хорошо проводящей сферической капли радиуса $a$ с тонким ДЭС толщины $d \ll a$ на ее поверхности. В результате полного решения этой обобщенной задачи Левича получается, что к потенциальному однородному потоку вне обтекаемой капли добавляется осесимметричное деформационное течение, а внутри нее, кроме сферического вихря Хилла [18], возникает еще и тороидальный вихрь Тейлора [16].

Безразмерный электрический потенциал $\Phi^{\prime}$ внутри капли $(R<1)$ и $\Phi$ вне $(R>1)$ в сферических координатах $(R, \theta, \varphi)$ задается соответственно формулами

$$
\begin{gathered}
\Phi^{\prime}=-\frac{2 q u_{0}}{\widetilde{\sigma}} R \cos \theta-\frac{2 q u_{1}}{\widetilde{\sigma}} R^{2} P_{2}(\cos \theta)+C^{\prime}, \\
\Phi=\left(-R+\frac{\gamma}{R^{2}}\right) \cos \theta+\frac{2 q u_{1}}{3 R^{3}} P_{2}(\cos \theta)+C,
\end{gathered}
$$

где $u_{0}$ и $u_{1}$ - соответственно характерные скорости ЭКД и ЭВТ на поверхности капли; разность констант $\left|C^{\prime}-C\right| \gg 1$ задает скачок потенциала поперек ДЭС; $\breve{\sigma}=\sigma^{\prime} / \sigma$ - отношение электропроводностей капли и окружающей среды; $q$ - поверхностная плотность заряда ДЭС; $\gamma=q u_{0}-1 / 2 ; P_{2}(\cos \theta)$ - полином Лежандра второй степени. В результате ЭКД разноименно заряженные обкладки двойного слоя приходят в движение, что превращает электростатический ДЭС в двойной токовый слой (ДТС) с антипараллельными конвективными поверхностными токами $j= \pm q u_{0} \sin \theta$, которые генерируют собственное азимутальное магнитное поле с индукцией $B=R \sin \theta\left(1+2 \gamma / R^{3}\right) / 2, B^{\prime}=q u_{0} R \sin \theta$ соответственно вне и внутри капли. Более того, внутри тонкого ДТС зажат простой магнитный слой типа поверхностной $\delta$-функции Дирака с азимутальной индукцией $B_{\Sigma}=-q u_{0} \delta(R-1) \sin \theta$. В результате на поверхности проводящей капли при ее ЭКД во внешнем электрическом поле возникает новая сложная векторная структура - тройной электромагнитный слой (ТЭМС) с взаимно перпендикулярными направлениями поверхностных меридиональных токов и азимутальным магнитным полем.

Переход от выражений (3) к суспензии капель основан на анализе полученного в классе обобщенных функций решения ЭКД для одной капли и последующего осреднения по ансамблю возможных конфигураций с помощью бинарной $g(R)$ коррелятивной функции, что обозначено угловыми скобками $\langle\ldots\rangle$. В итоге [19] получается система ЭГД-уравнений для суспензии таких капель с концентрацией $c<0.2$

$$
\left\{\begin{array}{l}
\operatorname{div}\langle\mathbf{u}\rangle=-3 c A_{2}\left[\frac{\mathrm{d} g}{\mathrm{~d} R}+g(2) \delta(R-2)\right] u_{\mathrm{e}} \cos \theta, \\
\operatorname{rot} \operatorname{rot}\langle\mathbf{u}\rangle+\nabla\langle p\rangle=6 c A_{1} g(R) \mathbf{u}_{\mathrm{e}} \\
\Delta\langle\Phi\rangle=3 c \gamma\left[\frac{\mathrm{d} g}{\mathrm{~d} R}+g(2) \delta(R-2)\right] \cos \theta
\end{array}\right.
$$

где $\mathbf{u}_{\mathrm{e}}$ - осредненная скорость ЭКД капель; $A_{1,2}=$ const . К этой системе надо добавить уравнения для центральной (пробной) капли и решить итоговую систему в трех областях: при $0<R<1$ внутри пробной капли, при $1<R<2$ в промежуточном слое и при $R>2$ во внешней 
области для эффективной среды, где находятся центры других капель. Решение уравнений для электрического потенциала с учетом системы (4) удобно искать в виде

$$
\begin{gathered}
\left\langle\Phi^{\prime}\right\rangle=\alpha^{\prime} R \cos \theta+C^{\prime}, \quad\langle\Phi\rangle=\left(\alpha R+\frac{\gamma}{R^{2}}\right) \cos \theta+C, \\
\left\langle\Phi_{*}\right\rangle=\left(\alpha_{*} R+\frac{\gamma_{*}}{R^{2}}+\frac{3 c \gamma}{R^{2}} \int_{2}^{R} x^{2}[g(x)-1] \mathrm{d} x\right) \cos \theta+C_{*},
\end{gathered}
$$

где постоянные $\alpha^{\prime}, \alpha, \alpha * \gamma, \gamma *$ определяются из граничных условий для полной системы осредненных ЭГД-уравнений. «Звездочка» в последнем выражении (5) и далее в этом разделе указывает на область $R>2$.

В работе [19] приведен вывод обобщенной формулы Максвелла для эффективной электропроводности $\sigma_{\mathrm{e}}$ однородной суспензии сферических капель с поверхностным зарядом тонкого ДЭС при их ЭКД, которая имеет вид

$$
\sigma_{\mathrm{e}}=\frac{\sigma\left[\alpha_{*}-c(\alpha+\gamma)\right]+\sigma^{\prime} c \alpha^{\prime}}{\alpha_{*}-c(\alpha+\gamma)+c \alpha^{\prime}}
$$

Числитель этой формулы соответствует среднеобъемной плотности тока в электрическом приближении $\langle\mathbf{j}\rangle_{V}=-\sigma\left(\nabla\left\langle\Phi_{*}\right\rangle-c \overline{\nabla\langle\Phi\rangle}\right)-\sigma^{\prime} c \overline{\nabla\left\langle\Phi^{\prime}\right\rangle}$, знаменатель - среднеобъемному значению градиента электрического потенциала $\nabla\langle\Phi\rangle_{V}=\nabla\left\langle\Phi_{*}\right\rangle-c \overline{\nabla\langle\Phi\rangle}+c \overline{\nabla\left\langle\Phi^{\prime}\right\rangle}$, где черта означает среднюю величину по объему пробной капли единичного радиуса.

Если в формуле (6) формально заменить все значения электропроводностей $\sigma_{\mathrm{e}}, \sigma^{\prime}, \sigma$ на соответствующие ДП $\varepsilon_{\mathrm{e}}, \varepsilon^{\prime}, \varepsilon$, то можно получить обобщение классической формулы Максвелла [20] для эффективной ДП $\varepsilon_{\mathrm{e}}$ однородной суспензии сферических капель с ДЭС при ее ЭКД, но только в электрическом приближении. Однако в электромагнитном приближении [15] при расчете среднеобъемной электрической индукции $\mathbf{D}=\varepsilon_{0}\left(\varepsilon_{1} \mathbf{E}+\left(\varepsilon_{1}-1\right)[\mathbf{u} \times \mathbf{B}]\right)$ нужно учесть ярко выраженную поверхностную, или $\Sigma$-фазу, образующую тонкий ТЭМС с взаимно ортогональными токами и магнитным полем. В итоге при расчете эффективной ДП суспензии капель с тонким ТЭМС $(d \ll a)$ при их ЭКД главным членом будет выражение

$$
\varepsilon_{\mathrm{e}} \approx \frac{c\left(\varepsilon_{\Sigma}-1\right) \overline{\left[\mathbf{u}_{\Sigma} \times \mathbf{B}_{\Sigma}\right]}}{\nabla\left\langle\Phi_{*}\right\rangle-c \overline{\nabla\langle\Phi\rangle}+c \overline{\nabla\left\langle\Phi^{\prime}\right\rangle}} \sim c\left(\varepsilon_{\Sigma}-1\right) q u_{0} u_{1} \frac{a}{d} \gg 1,
$$

где $\varepsilon_{\Sigma}$ - относительная ДП поляризованной среды внутри тонкого ДЭС, а прямая черта над векторным произведением $\left[\mathbf{u}_{\Sigma} \times \mathbf{B}_{\Sigma}\right]$ теперь означает среднее по объему тонкого шарового слоя, соответствующего ТЭМС.

Высокие значения $\mathbf{E}$ и $\varepsilon_{\mathrm{e}}$ обеспечивают снижение ЭГД-давления $p_{\mathrm{e}}$ под центром гро-

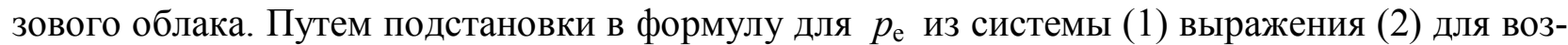
мущений АЭП при $N \sim 10^{3} \div 10^{4}$ и формулы (7) для $\varepsilon_{\mathrm{e}}$ с учетом ЭГДП можно получить, что в нижней части грозового облака давление может упасть на $3 \div 7 \%$ от атмосферного давления на соответствующей высоте. Это создает благоприятные условия для ускоренной реализации гидродинамической неустойчивости Рэлея-Тейлора [21]. Для дипольного грозового облака существуют два возможных сценария развития такой неустойчивости за счет ЭГДП и сильных возмущений АЭП: изменение зарядовой структуры на трипольную с возможным образованием воронок торнадо или струйный низовой прорыв [14] тяжелой и заряженной газокапельной среды дипольного облака, который обычно отличается от торнадо меньшей завихренностью при большем поперечном размере, а также наличием ярко выраженного расходящегося потока (всплеска) вблизи земной поверхности. В статье [13] получено решение задачи 
о движении к земной поверхности прорыва параболоидальной формы с уравнением границы $\left(r^{2}+z^{2}\right)-(z+2 b)=0$, где $b=\mathrm{const}$, которое можно выразить через безразмерные гидродинамическую $\psi_{*}$ и электрическую $\Psi_{\mathrm{e}}^{*}$ функции тока

$$
\psi_{*}=\frac{r_{*}^{2}}{2}-b_{*}\left(z_{*}+\sqrt{r_{*}^{2}+z_{*}^{2}}\right), \quad \Psi_{\mathrm{e}}^{*}=\frac{r_{*}^{2}}{2}-b_{*}\left(z_{*}+\sqrt{r_{*}^{2}+z_{*}^{2}}\right)+\Psi_{1}^{*},
$$

где $\Psi_{1}^{*}$ находится итерационным методом, описанном в [22]. Вблизи границы прорыва напряженность АЭП может возрастать в $5 \div 7$ раз [13] по сравнению с полем на удалении от Hee.

Трипольная структура заряда материнского грозового облака создает благоприятные условия для формирования воронки торнадо. Это обусловлено двумя важными электромагнитными факторами. Во-первых, на стадии «перезарядки» материнского облака [6] из дипольного в трипольное имеет место взаимодействие радиального тока $\mathbf{j}_{R}$ положительных кластерных ионов с вертикальной компонентой $\mathbf{B}_{z}$ геомагнитного поля. Следовательно, азимутальная магнитная сила $\mathbf{j}_{R} \times \mathbf{B}_{z}$ наряду с кориолисовой генерирует завихренность в материнском облаке, превращая его в торнадо-циклон. В дипольном облаке описанный механизм не действует, поэтому завихренность у низовых прорывов обычно составляет $10^{-2} \mathrm{c}^{-1}$, тогда как у торнадо она может быть выше $10 \mathrm{c}^{-1}$.

Во-вторых, сильные возмущения АЭП под облаком в совокупности с ЭГДП обеспечивают падение ЭГД-давления $p_{\text {e }}$ на определенном расстоянии до оси потока. Именно в области локального понижения $p_{\text {e }}$ должна формироваться воронка торнадо. Часто опусканию воронки могут сопутствовать некоторые специфические черты и даже аномальные явления $[2,12]$ такие, как «воротник», т.е. выступ квазицилиндрической формы в ее основании, или так называемые «рога дьявола» - две (или более) воронки, образующиеся из углов широкого вращающегося «воротника». Появление этих эффектов связано с особым распределением АЭП под грозовым облаком на разных этапах формирования трипольной структуры. Как видно из рис. 1, на начальном этапе ее образования ( $\beta=0.85$ в формуле (2)) профили АЭП

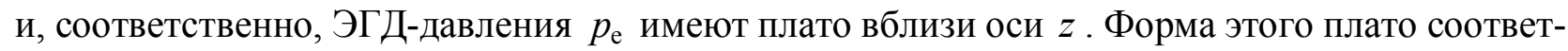
ствует конфигурации «воротника» (рис. 2,a), следовательно, могут возникать условия для образования последнего. В начале появления зоны повторного реверса АЭП вблизи оси об-

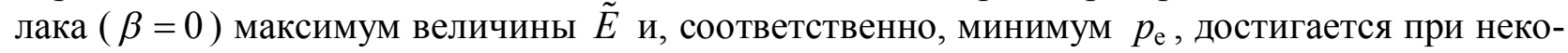
тором $\tilde{r}=\tilde{r}_{m} \neq 0$. В подобных случаях могут возникать «рога дьявола», которые иногда наблюдаются и при наличии центральной воронки, достигшей земной поверхности [2]. За счет начальной закрутки грозового облака две или более воронки могут вблизи земной поверхности сливаться в единый «мульти-торнадо» с многовихревой структурой потока (рис. 2, б).

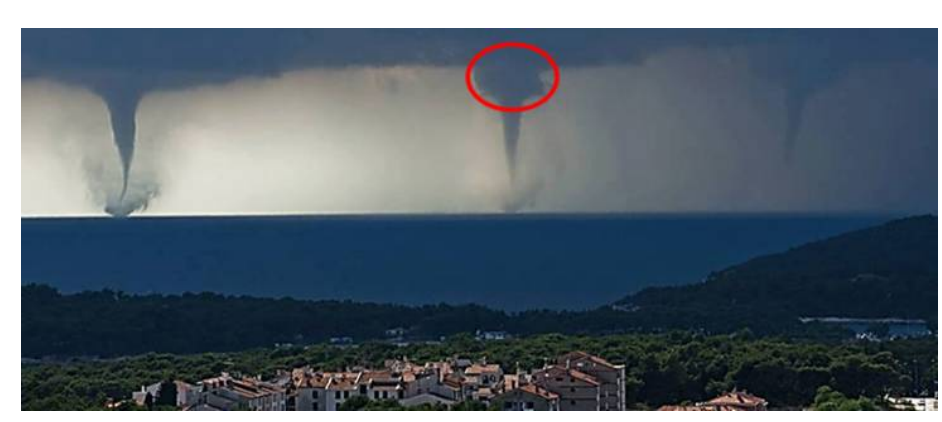

$a$

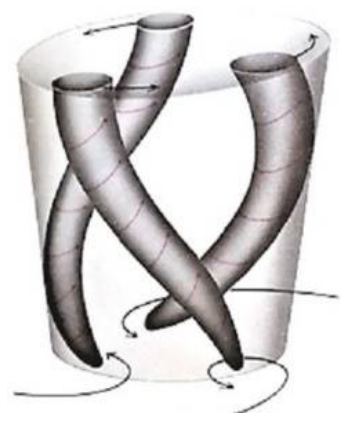

6

Рис. 2. $a$ - «воротник» (отмечен кружком) в основании воронки смерча в г. Пула, Хорватия, 2013 г., https://img.d3.ru/troinoe-tornado-v-khorvatii-455632/?sorting=rating; б - многовихревая структура потока в «мульти-торнадо» (из [23]) 
В статье [23] отмечается, что «мульти-торнадо» часто обладают большой разрушительной силой. Подобные торнадо наблюдались в городе Краснозаводске Московской области (3 июня 2009 г.) и в городе Джоплин, штат Миссури, США (22 мая 2011 г.). Максимальная скорость ветра в смерчах составляла порядка 90 м/с; в Краснозаводске пострадало 65 человек, а торнадо в Джоплине унес более 150 человеческих жизней и стал седьмым по числу жертв за всю историю наблюдений торнадо в США.

ЭГДП проявляется не только внизу центральной части ТЦ, но и (часто не менее сильно) вблизи подстилающей морской поверхности под центром облака, где возмущения АЭП наиболее значительные, а концентрация микрокапель достаточно высокая за счет брызгообразования из-за столкновения и обрушения поверхностных волн. В этой области падает ЭГДдавление за счет высоких значений $E$ и $\varepsilon_{\mathrm{e}}$ в (2) и (7), а при определенных $\beta$ (например, $\beta=-1)$ также радиальная сила $\mathbf{F}_{\mathrm{e}} \sim \nabla E^{2}$ принимает большие отрицательные значения (рис. 3). Эта сила в совокупности с вертикальной силой Кулона может приводить к образованию каскада из брызг и пены [1] над неустойчивым холмом приподнятой воды, двигающимся вслед за облаком. Иногда каскад формируется задолго до касания воронки смерча с подстилающей поверхностью, что было известно еще М.В. Ломоносову и А. Пуанкаре и описано в монографии [2], а в [1] представлена простейшая модель этого явления без учета ЭГДП.

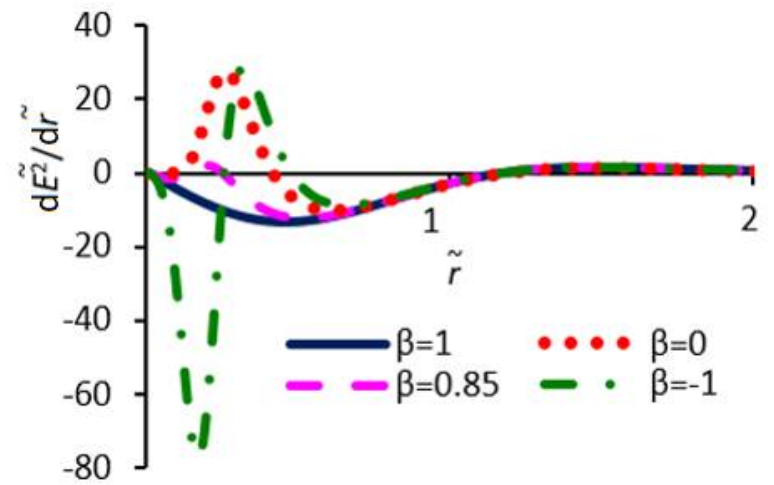

Рис. 3. Графики $\mathrm{d} \tilde{E}^{2} / \mathrm{d} \tilde{r}$ по формуле (2) при $N=4$ и различных значениях $\beta$

К дополнительным электромагнитным факторам генерации торнадо можно отнести различие магнитных свойств атмосферного азота и кислорода. При иногда наблюдаемых противоположных направлениях закрутки $[2,24]$ положительно заряженного нижнего центра ТЦ и его отрицательной периферии возникающие в грозовом облаке кольцевые токи генерируют магнитные поля. В результате в центральную зону ТЦ втягивается парамагнитный кислород, а диамагнитный азот выталкивается на периферию, что способствует увеличению массовой плотности столба воздуха у центра грозового облака и может благоприятствовать генерации торнадо. При однонаправленной закрутке центра и периферии облака магнитные поля кольцевых токов частично компенсируют друг друга, и в этом случае различие магнитных свойств кислорода и азота играет скорее стабилизирующую или ослабляющую роль.

\section{3. Заключение}

ЭГДП в грозовом облаке как суспензии микрокапель воды в совокупности с сильными возмущениями АЭП приводит к снижению ЭГД давления под центром облака. Это создает благоприятные условия для формирования торнадо и струйных низовых прорывов в зависимости от топологии зарядовой структуры грозового облака. Другие электромагнитные факторы играют вспомогательную или ослабляющую роль в зависимости от конкретных условий типа возможной сепарации парамагнитного кислорода и диамагнитного азота при разно- или однонаправленной закрутке в центре и на периферии торнадо-циклона. 


\section{Литература}

1. Натяганов В.Л., Маслов С.А. Ломоносов и загадки природного электричества. Часть 4. Электромагнитные механизмы формирования торнадоподобного смерча // Вестн. Моск. ун-та. Матем. Механ. 2014. № 2. С. 31-37.

2. Наливкин Д.В. Ураганы, бури и смерчи. Географические особенности и геологическая деятельность. Л.: Наука, 1969. 487 с.

3. Vonnegut B. Electrical theory of tornadoes // J. Geophys. Res. 1960. Vol. 65. Pp. 203-212.

4. Синкевич, О.А., Маслов, С.А., Гусейн-заде, Н.Г. Электрические разряды и их роль в генерации вихрей // Физика плазмы. 2017. Т. 43. № 2. С. 203-226.

5. Электровихревые течения / Под ред. Э.В. Щербинина. Рига: Зинатне, 1985. 315 с.

6. Маслов С.А. Электрические механизмы усиления завихренности в воронке торнадо. Вестн. Моск. ун-та. Матем. Механ. 2015. № 6. С. 54-58.

7. Маслов С.А. Влияние атмосферного электрического поля под грозовым облаком на формирование воронки торнадо. Вестн. Моск. ун-та. Матем. Механ. 2017. № 1. С. 57-61.

8. Френкель Я.И. Теория явлений атмосферного электричества. М.; Л.: ГИТТЛ, 1947. 152 с.

9. Williams E.R. The tripole structure of thunderstorms // J. Geophys. Res. D. 1989. V. 94. Pp. 1315113167.

10. Фейнман Р., Лейтон Р., Сэндс М. Фейнмановские лекции по физике. Т. 5. Электричество и магнетизм. М.: Мир, 1966. 296 с.

11. Саранин В.А. Устойчивость равновесия, зарядка, конвекция и взаимодействие жидких масс в электрических полях. М.; Ижевск: РХД, 2009. 331 с.

12. Маслов С.А., Натяганов В.Л. Влияние электрической структуры грозовых облаков на формирование торнадоподобных вихрей // Прикладная физика. 2015. № 6. С. 16-20.

13. Maslov S., Natyaganov V. Role of electromagnetic mechanisms in downburst and tornado formation. Physical and Mathematical Modeling of Earth and Environment Processes. Proc. 4th Int. Scientific School for Young Scientists, Ishlinskii Institute for Problems in Mechanics of Russian Academy of Sciences. Springer Nature Switzerland AG. 2019. Pp. 344-358.

14. Fujita T.T. Tornadoes and downbursts in the context of generalized planetary scales // J. Atmos. Sci. 1981. Vol. 38. Pp. 1511-1534.

15. Натяганов В.Л. Условность границ ЭГД и МГД приближений в некоторых задачах электромагнитной гидродинамики. Сб. докл. IX Международной научной конференции «Современные проблемы электрофизики и электрогидродинамики жидкостей», 25 - 28 июня 2009 г., СанктПетербург. СПб.: СОЛО, 2009. С. 131-134.

16. Натяганов В.Л. Электрокапиллярновихревая модель сферического вихря Хилла - Тейлора // Доклады академии наук. 2001. Т. 381. № 1. С. 50-52.

17. Левич В.Г. Физико-химическая гидродинамика. М.: ГИТТЛ, 1959. 700 с.

18. Бэтчелор Дж. Введение в динамику жидкостей. М.: Мир, 1973. 792 с.

19. Натяганов В.Л., Орешина И.В. Электрогидродинамика монодисперсных эмульсий // Коллоидный журнал. 2000. Т. 62. № 1. С. 90-100.

20. Ландау Л.Д., Лифшиц Е.М. Курс теоретической физики. Т. 8. Электродинамика сплошных сред. М.: Наука, 1982. 736 с.

21. Гендугов В.М., Натяганов В.Л., Чайка А.А. Косой удар цилиндрической струи о плоскость // Докл. Акад. наук. 2010. Т. 433. № 4. С. 481-484.

22. Вабищевич П.Н., Пулатов П.А. Численное решение внешней задачи Неймана // Журнал вычисл. матем. и матем. физ. 1987. Т. 27. № 4. С. $536-543$.

23. Snow J.T. The tornado // Scientific American. 1984. Vol. 250. Pp. 86-96.

24. Абрамов В.А. Смерчи над Владивостоком // Вестник ДВО РАН. 1998. № 2. С. 3-22. 